\title{
Estratégia\&Negócios
}

ISSN 1984-3372

http://www.portaldeperiodicos.unisul.br/index.php/EeN/

\section{ASPECTOS METODOLÓGICOS DOS ESTUDOS EM CAPITAL SOCIAL: UMA ANÁLISE DOS PRINCIPAIS PERIÓDICOS INTERNACIONAIS}

\section{METHODOLOGY ASPECTS OF SOCIAL CAPITAL STUDIES: ANALYSIS OF MAIN INTERNATIONAL JOURNALS}

\section{Rosana da Rosa Portella Tondolo}

Professora dos Cursos de Ciências Contábeis de Administração da Universidade do Vale do Rio dos Sinos - UNISINOS. Doutoranda do Programa de Pós-graduação em Administração da UNISINOS.

E-mail: rosanatondolo@gmail.com

\section{Vilmar Antonio Gonçalves Tondolo}

Professor do Programa de Pós-graduação da Universidade do Sul de Santa Catarina - UNISUL.

E-mail: vtondolo@gmail.com

\section{Cláudia Cristina Bitencour}

Professora do Programa de Pós-graduação em Administração da Universidade do Vale do Rio dos Sinos - UNISINOS. E-mail: claucbitencourt@gmail.com

Recebido em 17/11/2011. Aprovado em 03/05/2012. Disponibilizado em 25/05/2012. Avaliado pelo Sistema double blind review

R. eletr. estrat. neg., Florianópolis, v.5, n.1, p. 110-136, jan./abr. 2012 http://portaldeperiodicos.unisul.br/index.php/EeN/index
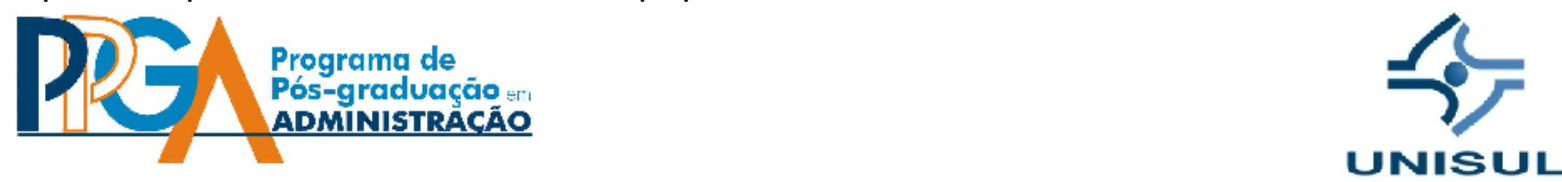

CCopyright 2008 UNISUL-PPGA/Estratégia e Negócios. Todos os direitos reservados. Permitida citação parcial, desde que identificada a fonte. Proibida a reprodução total. Em caso de dúvidas, consulte o editor: ademar.unisul@gmail.com; (48) 3229-1932. 


\section{RESUMO}

De acordo com a teoria sobre capital social, na medida em que os indivíduos interagem, o capital social tende a aumentar. Nesse sentido, o capital social pode ser visto como um estimulador/propulsor de resultados, tanto no campo da Administração quanto no campo da Sociologia ou, ainda, no terceiro setor. Nessa lógica, este estudo tem como objetivo investigar os aspectos metodológicos dos artigos publicados nos principais periódicos internacionais que abordam o tema capital social abrangendo as áreas de Administração, Sociologia e terceiro setor. Dessa forma, este estudo apresenta uma revisão bibliográfica sobre o tema capital social, além de analisar 20 estudos teórico-empíricos publicados nos principais periódicos internacionais entre os anos de 2000 e 2009. Para a realização desta investigação, foram seguidas as seguintes etapas metodológicas: (i) a consulta a quatro especialistas do tema, visando a identificar os principais periódicos internacionais que abordam o tema, ocasião em que foram identificados nove periódicos; (ii) classificação dos artigos identificados no período de 2000 a 2009, utilizando como critério de seleção a busca do termo "Social Capital" no título e resumo dos periódicos analisados; (iii) seleção dos artigos considerando os critérios de acessibilidade, e quanto à realização de um estudo teórico-empírico. Assim, resultando finalmente em uma amostra de 20 artigos, os quais são oriundos de sete dos periódicos analisados. Como principais resultados, apresentam-se: a predominância da abordagem quantitativa e o alinhamento metodológico existente entre os artigos publicados no mesmo periódico. Ao final, apresentaram-se algumas sugestões de estudos futuros.

Palavras-chave: Capital Social. Periódicos Internacionais. Aspectos Metodológicos. 


\section{INTRODUÇÃO}

O tema Capital Social apresenta distintas origens e atualmente é explorado em diferentes enfoques e por diversas áreas de pesquisa (PORTES, 1998; 2000). No entanto, o capital social pode ser originado a partir de três fontes distintas. A primeira apresenta o capital social a partir do controle social; a segunda, a partir dos benefícios mediados pela família; e a terceira; a partir dos recursos mediados por redes constituídas por membros ou indivíduos não familiares (PORTES, 1998).

Por mais que o termo capital social tenha sido originado na Sociologia, a partir dos estudos de Bourdieu e Coleman, o conceito de capital social passou a ser aplicado aos estudos organizacionais e está ganhando volume (ADLER; KWON, 2002). Adler e Know (2002) apresentam em seu estudo uma relação de pesquisas anteriores na área organizacional as quais se relacionaram ao tema capital social. Algumas das relações exploradas pelos autores permeiam o capital social associado a: aspectos de recursos humanos; comportamento organizacional; processos gerenciais e industriais; e processos estratégicos. (ADLER; KWON, 2002).

Nesse sentido, este artigo tem como objetivo investigar os aspectos metodológicos dos artigos publicados nos principais periódicos internacionais que abordam o tema capital social abrangendo as áreas de Administração, Sociologia e terceiro setor. Para isso, este estudo apresenta a análise realizada em sete periódicos internacionais que tratam o tema capital social. Estas fontes foram escolhidas com base na opinião de especialistas na área e considerando a temática a ser abordada por este artigo, a qual trata da abordagem do capital social em três perspectivas, sociológicas, administrativas e do terceiro setor. Dessa forma, foram analisados os seguintes periódicos internacionais: Academy of Management Journal; Administrative Science Quarterly; Journal of Management Studies; Nonprofit and Voluntary Sector Quarterly; Organization Studies; Sociological Forum; e, Strategic Management Journal. 


\section{CAPITAL SOCIAL}

O capital social pode ter três origens ou abordagens distintas (BARON; FIELD; SCHULLER 2000). A primeira é referente aos autores das ciências sociais, os quais criaram e difundiram o termo, entre eles destacam-se James Coleman (1988), Pierre Bourdieu (1980) e Robert Putnam (1995). A segunda destaca os elementoschave que compõem o capital social: confiança, coesão social, redes, normas e instituições, dentre os quais se destacam Portes (1998) e Nahapiet e Ghoshal (1998). A terceira, por sua vez, inclui atores que remontam elementos do capital social em suas obras, destacando-se Émile Durkheim e Max Weber (BARON; FIELD; SCHULLER, 2000).

O tema capital social é abordado desde o início da década de 1980, período no qual foram exploradas associações com diferentes áreas de conhecimento e contextos. Nessa temática, destacam-se alguns estudos seminais, os quais servem de base e inspiração para muitos trabalhos, dentre estes podem ser citados os estudos de Bourdieu (1980; 2003), Coleman (1988), Putnam (1995), Nahapiet e Ghoshal (1998), e Adler e Kwon (2002).

O uso do termo capital social foi introduzido por Pierre Bourdieu, quando ele se referia às vantagens e oportunidades de pertencer a certas comunidades (PORTES, 2000; ALBAGLI; MACIEL, 2002). Sendo seguido por James Coleman, o qual centrou sua investigação em indivíduos ou pequenos grupos como unidade de análise. Mesmo apresentando significantes variações entre os estudos, ambos os autores abordaram os benefícios resultantes aos indivíduos ou às famílias pela virtude de seus laços com terceiros (PORTES, 2000).

A partir dos estudos de Bourdieu (1980) e Coleman (1988), muitos outros estudos foram realizados enfocando o capital social em diferentes áreas, deixando, assim, de ser um tema específico da área de Sociologia e penetrando em diversas áreas (PORTES, 1998; 2000). Essa disseminação de estudos originou diversas associações com outros enfoques do capital social, gerando a diversidade de 


\section{ASPECTOS METODOLÓGICOS DOS ESTUDOS EM CAPITAL SOCIAL: UMA ANÁLISE DOS PRINCIPAIS PERIÓDICOS INTERNACIONAIS \\ Rosana da Rosa Portella Tondolo - Vilmar Antonio Gonçalves Tondolo - Cláudia Cristina Bitencour}

conceituações e definições relacionadas ao tema. A seguir, são apresentadas no Quadro 01 as principais definições de capital social abordadas pelos autores, as quais, como pode ser observado, elencam elementos como: recursos, estruturas sociais, indivíduos, oportunidades, normas, confiança e objetivos.

Diante disso, Bourdieu (2003) defende que o capital social é diferente das outras formas de capital, aumentando com o uso. A manutenção e o crescimento do capital social ocorrem devido à interação existente entre os atores que compõem uma rede. No entanto, Coleman (1988) entende o capital social como sendo semelhante ao capital humano e ao capital físico, pois o capital social não é completamente fungível, podendo ser específico para certas atividades. Assim como uma dada forma de capital social pode ser valiosa e facilitar certas ações, pode também ser desnecessária e compensar prejuízos de outras ações (COLEMAN, 1988).

Quadro 01 - Conceitos de Capital Social

\begin{tabular}{|l|l|}
\hline Conceitos & Autores \\
\hline $\begin{array}{l}\text { Capital social é o agregado do atual ou potencial recurso que é reunido para } \\
\text { possessão de uma durável rede de relações mais ou menos institucionalizadas de } \\
\text { mútuo conhecimento ou identificação. }\end{array}$ & Bourdieu (1985) \\
\hline $\begin{array}{l}\text { Capital social é uma variedade de entidades com dois elementos em comum, os } \\
\text { quais consistem de algum aspecto de estruturas sociais e facilitam evidentes ações } \\
\text { de atores (pessoas ou corporações) com a estrutura. }\end{array}$ & Coleman (1988) \\
\hline $\begin{array}{l}\text { Capital social é um recurso que deriva de atores de específicas estruturas sociais, } \\
\text { sendo usado para perseguir seus interesses, é criado pelas trocas nas relações entre } \\
\text { atores. }\end{array}$ & Baker (1990) \\
\hline $\begin{array}{l}\text { Capital social é o conjunto de elementos das estruturas sociais que afetam as } \\
\text { relações entre pessoas e são inputs ou argumentos da produção e/ou utilidade } \\
\text { funcional. }\end{array}$ & Schiff (1992) \\
\hline $\begin{array}{l}\text { Capital social é como amigos, colegas e, de forma mais geral, contatos diretos de } \\
\text { quem você recebe oportunidades para usar seu capital humano e financeiro. }\end{array}$ & Burt (1992) \\
\hline $\begin{array}{l}\text { Capital social são traços da vida social - redes de contatos, normas e confiança - } \\
\text { que possibilitam aos participantes agirem juntos mais efetivamente para perseguir } \\
\text { objetivos em comum. }\end{array}$ & Putnam (1995) \\
\hline
\end{tabular}

Fonte: Adaptado de Portes (1998); Walker (2008). 
Conforme Walker (2008), Putnam surge com um estudo sucessor à Coleman, uma vez que ambos apresentam uma visão conceitual muito próxima. Contudo, é importante ressaltar que, enquanto Coleman busca explicar as consequências das relações sociais, Putnam preocupa-se em buscar formas de melhorar a saúde política da sociedade. Porém, ambos dão prioridade ao desempenho das normas e possuem um forte compromisso com valores coletivos, questionando, assim, o valor do capital humano, quando dissociado de relações sociais amplas, discordando de abordagens mais ortodoxas, as quais defendem o individualismo e a racionalidade (ALBAGLI; MACIEL, 2002).

Putnam (1995) evidencia, em seu estudo, as relações sociais e a presença de confiança e de cooperação como fontes de mútuos benefícios. Consoante isso, Nahapiet e Ghoshal (1998) defendem a existência de três dimensões no capital social (estrutural, cognitiva e relacional) e que a dimensão relacional, a qual mede confiança, normas, obrigações e identificação social, cresce nas redes em várias direções, com a presença de elos fortes e recíprocos.

De acordo com Albagli e Maciel (2002), dois dos pressupostos utilizados por Putnam, em sua definição de capital social (redes, normas e confiança), são implícitos em sua conceituação. Isso se deve ao fato de que as redes e normas estão empiricamente associadas e têm efeitos econômicos significativos para a comunidade, surgindo uma função instrumental para o capital social. Já a confiança é alcançada quando há conhecimento mútuo entre os membros de uma comunidade e uma forte tradição de ação comunitária.

Segundo Sarate e Macke (2007), o capital social possui fontes inesgotáveis, desde que sejam utilizadas, mas podem diminuir e se tornarem escassas se não forem utilizadas. Esses autores defendem que o capital social reside nas relações, não sendo propriedade exclusiva de indivíduos. Por esse motivo, o desenvolvimento do capital social é afetado significativamente por fatores que estruturam a evolução das relações sociais. 
$\mathrm{Na}$ busca pelo entendimento do capital social, há pelo menos três dimensões sugeridas na literatura: estrutural, cognitiva e relacional. A dimensão estrutural está relacionada ao sistema de relações e aos links existentes entre pessoas ou unidades, enquanto que a dimensão cognitiva se refere aos recursos que são providos por representações, interpretações e sistemas de significado, os quais são associados às partes e, por fim, a dimensão relacional descreve a espécie de relações individuais desenvolvidas entre os indivíduos (NAHAPIET; GHOSHAL, 1998). Nesse contexto, Sarate e Macke (2007) buscaram apresentar as dimensões e os elementos do capital social, os quais foram abordados por Nahapiet e Ghoshal (1998), e se apresentam no Quadro 02.

Quadro 02 - Dimensões do capital social

\begin{tabular}{|l|l|l|}
\hline Dimensão Estrutural & Dimensão Cognitiva & Dimensão Relacional \\
- Conexões da rede; & - Códigos e linguagem & - Confiança; \\
- Configuração da rede; & compartilhados; & - Normas; \\
- Adequação da & - Narrativas compartilhadas. & - Obrigações e expectativas; \\
organização. & & - Identificação social. \\
\hline
\end{tabular}

Fonte: Sarate e Macke (2007, p. 3) adaptado de Nahapiet e Ghoshal (1998).

\section{METODOLOGIA}

A fim de atender ao objetivo deste estudo, foram consultados quatro especialistas da área de estudos organizacionais, visando a identificar os principais periódicos internacionais da referida área, os quais abordam o tema. Nessa etapa, foram identificados nove periódicos (Academy of Management Review; Academy of Management Journal; Administrative Science Quarterly; Annual Review of Sociology; Journal of Management Studies; Nonprofit and Voluntary Sector Quarterly; Organization Studies; Sociological Forum; e Strategic Management Journal).

Nestes periódicos, foram identificados inicialmente 89 artigos no período de 2000 a 2009, utilizando-se como critério de seleção a busca do termo "Social Capital" no título e resumo dos periódicos analisados. Em seguida, estes 89 estudos 
foram selecionados considerando os critérios de acessibilidade e quanto à realização de um estudo teórico-empírico, exploratório, com abordagem qualitativa. Resultando finalmente em uma amostra, não probabilística, de 20 artigos, os quais são oriundos de sete dos periódicos analisados.

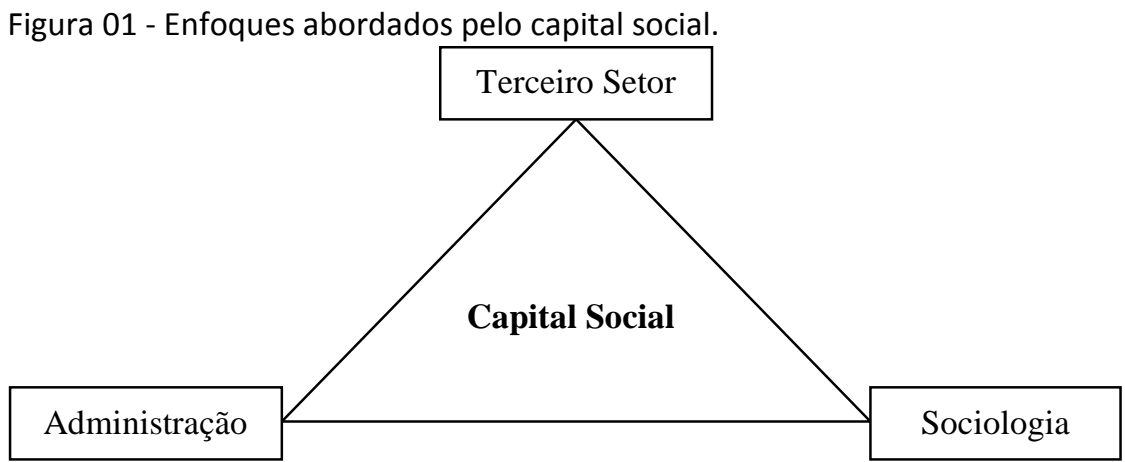

Fonte: Elaborado pelos autores, 2012.

A escolha destes periódicos utilizou como base os três enfoques do capital social: o aspecto sociológico do capital social, sendo esta a origem do tema; o aspecto da administração, sendo a base teórica a que este artigo está vinculado; e o aspecto do terceiro setor ligado ao tema de interesse dos pesquisadores, como pode ser observado na Figura 01.

Nesta amostra de artigos, foram verificados os aspectos metodológicos, assim foi analisado o objetivo do estudo e o objeto analisado por cada um dos artigos. Também foram observadas algumas características presentes nesses estudos, tais como: tamanho da amostra, abordagem, técnicas de coleta e análise dos dados, as quais foram consideradas como categorias de análise, seguindo a lógica da análise de conteúdo. 


\section{ANÁLISE METODOLÓGICA DOS ARTIGOS DOS PERIÓDICOS INTERNACIONAIS}

Esta seção apresenta a análise metodológica de 20 artigos, os quais são originados de sete periódicos internacionais. Os artigos são apresentados e analisados de forma ordenada por autor e periódico, respectivamente:

- quatro artigos do Academy of Management Journal;

- dois artigos do Administrative Science Quarterly;

- três artigos do Journal of Management Studies;

- quatro artigos do Nonprofit and Voluntary Sector Quarterly;

- dois artigos do Organization Studies;

- dois artigos do Sociological Forum;

- e três artigos do Strategic Management Journal.

Dentre os artigos destaca-se o estudo de Lester et al. (2008), no qual foram analisados os senadores, deputados e secretários dos gabinetes presidenciais americanos que deixaram seus cargos no período de 1988 a 2003. Os secretários dos gabinetes individuais foram identificados por cada um dos 14 sites do departamento de gabinetes, enquanto que os senadores foram identificados pelo sistema de dados do Congresso Nacional dos Estados Unidos (Lexis-Nexis). A amostra utilizada consiste em 66 secretários de gabinetes, 74 senadores e 96 deputados, no total de 236 indivíduos. O número de secretários e senadores englobam toda a população, enquanto que a população de deputados era de 433 durante o período analisado. Os autores desenvolveram uma pesquisa quantitativa, a qual verificou inicialmente a média, o desvio padrão e a correlação das variáveis examinadas, depois foi construída uma categorização dessas variáveis e, posteriormente, foi realizada uma análise de regressão para teste de dois modelos. O objetivo dos autores foi explorar a profundidade, a amplitude e a deterioração do capital humano e social dos ex- 
funcionários de alto escalão do governo e achar que essas dimensões do capital humano e social são preditores influentes da diretoria das empresas fora do governo.

Já Somaya, Williamson e Lorinkova (2008) analisaram as principais empresas inovativas constantes no ranking Fortune 500, as quais utilizava escritórios de advogados especialistas em processos de patentes no período de 1991 à 1995. A unidade de observação foram as empresas-pares por ano. Os autores focaram nos processos de patente por entenderem que o capital humano e social, nesses processos, é um importante recurso para a competição na indústria e que o movimento dos colaboradores de uma empresa para outr, pode causar imapcto na performance das empresas. A amostra utilizada foi de 109 empresas, com base na Fortune 500, das quais 29 são do setor químico, 19 são indústrias de computadores, 38 são do setor de eletrônicos, 9 são do ramo farmacêutico, e 14 são de equipamentos científicos e fotográficos. Essas empresas foram escolhidas por serem clientes potenciais de escritórios de advogados especialistas em processos de patentes, dos quais 123 fizeram parte da amostra. Os autores desenvolveram uma pesquisa quantitativa, a qual apresentou estatística descritiva e as correlações entre as variáveis analisadas, posteriormente foi aplicado a análise de regressão de Poisson, testando sete modelos. O objetivo dos autores foi explorar como a mobilidade entre os competidores e a mobilidade entre os potenciais cooperadores são diferentes; para isso, os autores utilizaram a teoria do capital social.

A pesquisa realizada por Stam e Elfring (2008) analisou 90 novos empreendimentos na indústria de software livre dos países baixos. Os autores utilizaram de múltiplas fontes de dados, a fim de aumentar a validade e credibilidade da pesquisa. Os primeiros dados foram coletados em 2005, por meio de uma survey. Também foram usados dados múltiplos secundários para construir uma lista inicial, a qual foi encontrada 127 empresas. Depois, foram conduzidas as entrevistas com os especialistas das empresas, a fim de validar a primeira lista, combinando, assim, as abordagens objetivas e cognitivas para idenficar as preferências da população estudada. Nove empreendimentos foram mencionados por mais de um especialista e 
foram adicionados à lista inicial, e, com as alterações no decorrer da pesquisa, a população final foi de 125 empresas. Foram conduzidas entrevistas curtas com 121 respondentes de 90 empresas. Os autores realizaram primeiramente uma análise estatística descritiva, uma correlação e um modelo hierárquico moderado da análise de regressão, o qual tinha como objetivo testar as hipóteses apresentadas pelo estudo. O objetivo dos autores foi examinar como a configuração dos laços das relações moldam-se entre a orientação empreendedora e a performance do novo empreendimento de um time fundador intra e extra setorial.

No estudo de Pil e Leana (2009), foi realizada uma survey com professores de escolas de Ensino Fundamental do Nordeste dos Estados Unidos. Essa pesquisa é parte de uma grande pesquisa, cujo objetivo inicial foi examinar como estava sendo implementado (scaled up) o novo currículo de matemática em escolas múltiplas. Nessa etapa, 199 escolas participaram, totalizando 5.205 professores respondentes. Além dessa etapa, os autores classificaram os respondentes em equipes e, finalmente, restringiram a amostra, eliminando as equipes que não apresentavam boa representação. Com isso, a amostra final totalizou 24.187 estudantes, 1013 professores e 239 graus de equipes. Os autores utilizaram a técnica de correlação para análise dos dados e, para testar as hipóteses, utilizaram o modelo de regressão linear hierárquica. O objetivo dos autores foi investigar os efeitos do capital humano e social dos professores na performance de crescimento dos estudantes.

Xiao e Tsui (2007) conduziram seu estudo no setor de Tecnologia da Informação na China. Primeiramente, os autores procuraram seis especialistas no setor para coletar sugestões de empresas a serem estudadas. Como base de amostragem, escolheram empresas de hardware, software, telecomunicações e provedores de internet que possuíam em torno de 1000 colaboradores. O passo seguinte ocorreu nos próximos seis meses, quando os autores identificaram 16 empresas, das quais tiveram acesso somente a quatro (Hardware, Software, Telecom e Mobile). Os autores realizaram uma survey com os colaboradores dessas empresas, considerando as áreas específicas de interesse. Também realizaram entrevistas com o 
executivo da área de Recursos Humanos de cada empresa e nove entrevistas com os gestores da Telecom e da Mobile. As técnicas para análise dos dados foram a estatística descritiva e análise de correlação. Os dados qualitativos corroboraram e enriqueceram a análise quantitativa, mas não está descrito no artigo como esses dados foram analisados.

Enquanto isso, Maurer e Ebers (2006) realizaram uma análise comparativa longitudinal de seis novas empresas alemãs de biotecnologia. Dessa forma, foi conduzido um estudo exploratório por meio da abordagem Grounded-theory. Foram realizadas entrevistas com especialistas do setor, a fim de verificar entre os atores possíveis proxies do sucesso biotecnológico. Foram coletadas diferentes evidências de dados a partir de entrevistas, visitas a sites e arquivos gravados, sendo realizadas 19 entrevistas semiestruturadas. O objetivo dos autores foi explorar como a configuração, gestão e evolução do empreendedorismo do capital social das empresas afetam o seu desempenho.

A pesquisa realizada por Wu (2008) teve como base amostral as indústrias chinesas instaladas em Hong Kong. Foi realizada uma amostra randômica dos participantes, os quais foram selecionados entre os membros diretores da Associação de Indústrias Chinesas de Hong Kong, uma organização bem conhecida. Os diretores forneceram informações sobre aproximadamente 3.700 membros (setor, localização, história, número de colaboradores, área de negócio e o nome do diretor dessas empresas). Foi realizada uma survey, com questionários estruturados em inglês e chinês. Todos os respondentes receberam ambos os questionários. Os questionários foram analisados por meio de uma escala likert de cinco pontos. Outras informações foram incluídas, tais como idade, educação, estado civil, posição atual na empresa, idade da empresa, tipo de setor e o tamanho da companhia. A survey foi realizada por e-mail, e obteve-se o retorno de 108 questionários completos. Em um segundo momento da pesquisa, foram realizadas entrevistas com 10 diretores de organizações que não estavam originalmente incluídos na amostra da survey, esses diretores responderam às mesmas questões que compuseram a survey. Para a análise dos 
dados, foi utilizada a estatística descritiva, a correlação do Pearson, utilizando-se do alfa de Cronbach para a validação dos dados. O objetivo do autor foi investigar a função da mediação do compartilhamento da informação nas relações entre as dimensões do capital social e a competitividade da firma.

Gopalakrishnan, Scillitoe e Santoro (2008) realizaram uma pesquisa quantitativa, por meio de uma survey com 184 alianças entre empresas, entre os anos de 1995-2000. Os autores utilizaram, como técnicas de análise dos dados, a estatística descritiva e a matriz de correlação, a qual foi aplicada em todas as variáveis examinadas neste estudo. Em um segundo momento, os autores aplicaram a técnica de análise de regressão hierárquica; assim, os autores criaram três modelos separados de regressão a fim de testar as hipóteses. O objetivo dos autores foi examinar os fatores que influenciam a extensão do capital financeiro das empresas de Biotecnologia quando estas conquistam uma aliança com empresas farmacêuticas, tendo como base de análise os aspectos da visão baseada em recursos e a teoria do capital social.

Enquanto isso, Stevenson e Radin (2009) buscaram descobrir como o conselho administrativo era constituído. Primeiramente por meio de um estudo direto do conselho e seus membros. Neste estudo, os autores escolheram a abordagem multi-métodos: em um primeiro momento, os autores conduziram uma survey com uma amostra de 14 empresas, a qual foi realizada com os membros do conselho e 14 diretores das empresas. Os autores conduziram entrevistas com os diretores, presidentes e membros do conselho a fim de complementar os resultados quantitativos. Para análise dos dados, os autores utilizaram o coeficiente de correlação intraclasse em um modelo linear hierárquico, por meio de uma ANOVA. Para verificação dos laços prioritários, considerando a posição dos respondentes, os autores utilizaram o modelo de regressão.

O estudo apresentado por Yeung (2004) se caracteriza por ser uma pesquisa quantitativa baseado em dados secundários, os quais são da pesquisa European Value Study (EVS). Os dados da EVS foram coletados por meio de uma 
survey e por meio de entrevistas com 1038 respondentes, entre os meses de setembro e outubro de 2000, sendo representativos a toda a Finlândia. O autor buscou classificar os dados da EVS em quatro grupos diferentes de voluntarismo: (i) pessoas que voluntariam somente em igrejas ou instituições religiosas; (ii) voluntários tanto em instituições religiosas como em outros contextos; (iii) pessoas que são voluntários fora do contexto da igreja; e (iv) pessoas que não praticam o voluntariado. Para isso, o autor se utilizou da questão número 5 do instrumento original de pesquisa utilizado pela EVS, a qual abordava o trabalho voluntário e possuía 15 tipos de organizações e atividades do trabalho voluntário. Para mensurar o capital social, a religiosidade e as variáveis sociodemográficas, foram utilizadas como modelos de análise as tabelas cruzadas, a ANOVA e a análise de regressão logística. A ANOVA identificou as diferenças entre os grupos analisados, e a significância dos resultados foi testada com o teste de Pearson qui-quadrado. O objetivo do autor foi contribuir para o avanço da literatura por meio da inclusão de diferentes formas de religiosidade e capital social.

Isham e Kolodinsky (2006) também utilizaram uma base de dados já existente. Os autores utilizaram, em seu artigo, a base de dados de uma survey anual realizada com adultos do estado de Vermont. Os dados foram coletados em 2002 e envolvem dados com capital social e organizações sem fins lucrativos. Para análise dos dados, foi utilizada a abordagem quantitativa por meio do modelo econométrico, no qual foram analisados 677 questionários completos. Com a análise realizada, foi possível distinguir entre a probabilidade de formação do capital social e o nível de formação do capital social.

No estudo de Graddy e Wang (2009), foi desenvolvido um modelo de determinantes para o desenvolvimento de fundações comunitárias. O objetivo do artigo foi realizar um teste empírico do seu modelo. Para isso, os autores necessitaram de dados em capital social, fundações comunitárias e seus competidores, bem como características da comunidade sociodemográfica. Os autores utilizaram uma base de dados secundária, cujos dados foram coletados em 
2000, pela Social Capital Community Benchmark Survey (SCCBS), e cuja coleta foi conduzida nacionalmente em 41 comunidades locais americanas. Assim, este estudo se utilizou dos dados da SCCBS que incluem 26.230 respondentes de 284 microrregiões (counties). Após os filtros e as classificações da amostra realizada pelos autores, a pesquisa contemplou a análise de 132 microrregiões. Para a análise dos dados, foi utilizada a abordagem quantitativa, por meio da análise estatísitica descritiva e uma regressão ordinária do quadrado mínimo.

Brown e Ferris (2007) também utilizaram uma base de dados já existente, cujos dados foram coletados em 2000 pela SCCBS (Social Capital Community Benchmark Survey), a qual possui uma amostra de 29.333 respondentes de 40 comunidades de todas as partes dos EUA. Nesse estudo, foi utilizada uma amostra de 3.003 respondentes, a qual foi entendida como representativa nacional. Os dados foram analisados de forma quantitativa: utilizou-se, primeiramente, a técnica de análise de correlação entre o capital social, doação e o voluntariado; posteriormente, utilizou-se a análise estatística descritiva; e finalmente aplicou-se o modelo de regressão. O objetivo dos autores foi examinar o impacto do capital social na filantropia.

No entanto, Crotty (2006) escolheu, como objeto de pesquisa, a região de Samara Oblast, na Rússia. Essa região foi escolhida porque foi uma região muito importante no período soviético, pois concentrava diferentes setores industriais e, após esse período, teve como diferencial a alta taxa de investidores externos. A base de dados inicial dos autores contemplou nove entrantes nesse mercado, e, com a ajuda da Universidade do Estado de Samara, seis destas empresas foram localizadas. A pesquisa caracteriza-se por sua abordagem qualitativa. A técnica de coleta de dados foi a entrevista, a partir de um instrumento previamente estruturado, tendo questões que serviam de guia. As entrevistas foram realizadas em russo e, em seguida, foram traduzidas e transcritas. Cada entrevista durou de 60 a 120 minutos. Para análise dos dados, foi adotado o método de triangulação, o qual se utilizou das entrevistas realizadas, dos materiais secundários coletados (incluindo reportagens oficiais e 
outros materiais impressos). Após a triangulação, cada entrevista foi codificada e foram identificados os temas-chave. Com a emergência dos temas, vários insights teóricos, incluindo o esquema oportunidade-motivação-habilidade (ADLER; KWON, 2002), foram revisitados a fim de clarificar e entender os dados acumulados. 0 objetivo dos autores foi realçar os motivos para a continuação da contração do espaço civil russo, ou seja, o comportamento do movimento ambiental na região de Sumara Oblast, na Rússia, utilizando-se da lente do capital social.

A pesquisa realizada por Batjargal (2003) caracteriza-se por sua abordagem quantitiva. A técnica de coleta de dados foi o questionário, na qual primeiramente foram questionados 75 empresários russos no ano de 1995; posteriormente, foram aplicados questionários adicionais com os 56 respondentes da primeira etapa, em 1999. Em 1994, foi realizada uma etapa-piloto com seis empresários de Moscou. Na amostra, estão incluídas três cidades russas e quatro diferentes setores. Para análise dos dados, os autores utilizaram a estatística descritiva, a correlação de Pearson e a regressão multivariada. O autor teve como objetivo examinar o impacto do capital social do empresário no desempenho das suas empresas na Rússia Pós-Soviética.

White e Glick (2000) também utilizaram uma base de dados já existente. A base de dados High School and Beyond Survey (HSBS), a qual contém dados longitudinais, é nacionalmente representativa e contém números substanciais de imigrantes e filhos de imigrantes. A pesquisa realizada começou em 1980, com os alunos que frequentavam o segundo ano do Ensino Médio. A pesquisa caracteriza-se pela abordagem quantitativa, sendo utilizado o modelo de regressão logística para a análise dos dados com os alunos que permaneciam no Ensino Médio em fevereiro de 1982. Também foi utilizada a regressão logística multinomial, a fim de modelar as atividades daqueles que não estavam no Ensino Médio em 1982, para determinar a probabilidade dos participantes em atividades que proporcionam ganhos econômicos imediatos. O objetivo dos autores foi investigar a diferença dos imigrantes e nativos em atividades de adolescentes dois anos depois deles estarem no Ensino Médio. 
Walker (2008) também utilizou a base de dados secundários da American Citizen Participation Study (ACPS), e sua pesquisa original analisou a relação entre a participação em sociedade civil e a atividade política institucionalizada. A pesquisa caracteriza-se pela abordagem quantitativa, sendo utilizado o modelo de equações estruturais. O objetivo do autor foi testar os efeitos da participação em associações voluntárias no engajamento cívico.

O estudo realizado por Acquaah (2007) coletou os dados com executivos seniors ou diretores, seus representantes e gestores da área financeira. A amostra consiste em 200 médias e grandes empresas selecionadas no Ghana Business Directory (2001) e membros da diretoria da Associação das Indústrias de Ghana. Os dados foram coletados entre os anos de 2001 e 2002. A pesquisa caracteriza-se pela abordagem quantitativa, sendo utilizadas a análise fatorial, a correlação bivariada e a regressão múltipla hierárquica. O objetivo do autor foi replicar e estender uma pesquisa anterior, realizada na China, a uma região emergente do sub-Saara Africano.

A pesquisa desenvolvida por Moran (2005) coletou dados de 120 gestores de produção e vendas do setor farmacêutico. A pesquisa caracteriza-se pela abordagem quantitativa, sendo utilizadas a estatística descritiva e a correlação bivariada, bem como uma análise de regressão multivariada foi utilizada para testar as hipóteses. O objetivo do autor é examinar o impacto do capital social dos gestores na performance gerencial. Para isso, foram comparadas duas dimensões do capital social, a dimensão estrutural e a dimensão relacional.

Já o estudo realizado por Luo (2003) utilizou duas fontes de dados: os dados de variáveis do setor foram extraídas do China Statistical Yearbook (CSY); e os dados das relações/redes de gestores foram oriundas de uma survey a qual foi realizada com os altos executivos de empresas chinesas, sendo realizada durante os anos de 1999 e 2000. A fim de validar e checar o instrumento da survey, foi conduzido um teste-piloto com 30 integrantes de um programa de trainee. Os dados foram coletados em 364 empresas na China. A pesquisa caracteriza-se pela abordagem quantitativa, sendo utilizada estatística descritiva, a correlação de Pearson, bem como 
uma análise de regressão multivariada foi utilizada para examinar como as redes de gestão são influenciadas pelas dinâmicas industriais que as empresas enfrentam. 0 objetivo do autor é analisar a forma como as condições industriais influenciam as relações gerenciais do nível de executivos com outras entidades, como compradores, fornecedores, concorrentes, distribuidores e reguladores em um mercado emergente. Tendo sido abordado individualmente cada estudo, na próxima seção, passa-se a discutir as metodologias utilizadas nos referidos estudos.

\section{DISCUSSÃO DOS DADOS}

Após a análise dos 20 artigos, pôde-se verificar a predominância da abordagem quantitativa, uma vez que essa abordagem foi empregada em $75 \%$ dos artigos analisados, enquanto $15 \%$ dos artigos apresentam abordagem qualiquantitativa e apenas $10 \%$ dos artigos apresentam abordagem qualitativa. Outro aspecto a ser destacado é que, para fins classificatórios, foi utilizada neste artigo a técnica de análise dos dados em uma visão geral, sem detalhar especificidades das técnicas aplicadas.

Quando analisados os artigos com abordagem qualitativa, pôde-se verificar que as técnicas utilizadas foram a grounded theory, isto é, o uso de entrevistas como método de coleta e a triangulação de dados para validação. Enquanto isso, as técnicas de análise mais utilizadas nos artigos quantitativos foram a correlação e o modelo de regressão, as quais foram empregadas em $65 \%$ dos artigos analisados. Outra técnica bastante utilizada foi a estatística descritiva, empregada em $55 \%$ dos artigos analisados. Ainda foi possível identificar outros tipos de técnicas, como análise fatorial, ANOVA, tabelas cruzadas, modelo econométrico e equação estrutural, utilizadas em apenas $5 \%$ dos artigos. Vale destacar que todos os artigos que apresentaram a abordagem quantitativa utilizaram mais de uma técnica de análise dos dados. 
Quando analisadas semelhanças entre os artigos publicados no mesmo periódico, foi possível verificar que todos os artigos analisados no Academy of Management Journal apresentam aspectos metodológicos semelhantes, uma vez que todos os artigos publicados possuem abordagem quantitativa e se utilizam de pelo menos duas técnicas de análise, as quais são correlação e regressão. Outra semelhança encontrada foi no periódico Nonprofit and Voluntary Sector Quarterly, em que todos os artigos analisados apresentam uma abordagem quantitativa, na qual os dados utilizados são oriundos de bases de dados secundários. Quanto aos demais periódicos, não foi observado um padrão semelhante.

\section{CONSIDERAÇÕES FINAIS}

Este estudo investigou os aspectos metodológicos dos artigos publicados nos principais periódicos internacionais que abordam o tema capital social abrangendo as áreas de Administração, Sociologia e terceiro setor. Para tal, foi realizada uma consulta com especialistas, ocasião em que foram definidos os principais periódicos internacionais que abordam o tema.

O objeto de estudo deste estudo são os artigos teórico-empíricos que abordam o tema capital social integrantes publicados nestes periódicos no período de 2000 a 2009. Dessa forma, constatou-se que o tema apresenta predominantemente a abordagem quantitativa, uma vez que está presente em $90 \%$ dos artigos analisados, considerando a junção dos artigos que apresentam abordagem quantitativa (75\%) com os artigos que apresentam abordagem quali-quantitativos (15\%).

Também foi possível verificar claramente o alinhamento existente nos periódicos Academy of Management Review e Nonprofit and Voluntary Sector Quarterly, os quais apresentam semelhanças entre os artigos analisados, as quais são relacionadas tanto a aspectos metodológicos, como abordagem metodológica, 
técnicas de coleta e análise dos dados, quanto a linha de autores utilizados e a construção das seções desses artigos. Como principal contribuição deste estudo, destaca-se a identificação dos métodos utilizados e a tendência de alinhamento dos periódicos, o que pode ser útil para estudos futuros sobre o tema.

Por fim, como limitações deste estudo, destaca-se o tamanho da amostra de artigos analisados, uma vez que se essa amostra fosse maior, poderia ser possível inferir mais relações entre os periódicos analisados e os aspectos metodológicos apresentados pelos artigos publicados nesses periódicos, limitando a possibilidade de generalização dos resultados. Sugere-se ainda a realização de estudos complementares que aprofundem e ampliem o escopo da análise realizada por este estudo e a quantidade de artigos analisados. Bem como, estudos que contribuam com o tema capital social, tendo estes uma abordagem teórico-empírica ou somente teórica, mas que apresentem contribuições significativas para as áreas, como, por exemplo, o terceiro setor.

\section{METHODOLOGY ASPECTS OF SOCIAL CAPITAL STUDIES: ANALYSIS OF MAIN INTERNATIONAL JOURNALS}

\section{ABSTRACT}

According to the social capital theory, the extent to which individuals interact among each other, social capital tends to increase. In this sense, social capital can be seen as a stimulator/driver of outcomes. Those outcomes are both in the field of administration, and in the field of sociology or third sector. In this sense, this study aims to investigate methodological aspects of the articles published in leading international journals, which address social capital, covering areas of administration, sociology and third sector. Thus, this study presents a literature review on the subject of social capital, reviewing 20 theoretical and empirical studies published in leading international journals between the years of 2000 to 2009. In order to carry out this research, we followed the following methodological steps: (i) consulting four experts on the 
subject, identifying the main international journals on the topic, at this stage were identified 09 journals, (ii) classification of articles identified in the period of 2000 to 2009, using as selection criteria of the search term "Social Capital" in the title and abstract of the journals analyzed, (iii) selection of articles considering the criteria of accessibility, as well as, the criteria of theoretical and empirical study. Subsequently, we got a sample of 20 articles, which were from 07 journals. The main results are presented: the dominance of the quantitative approach and alignment between the methodological papers published in each journal individually. At the end, we presented some suggestions for further studies.

Key-Words: Social Capital. International Journals. Methodology Aspects.

\section{REFERÊNCIAS}

ACQUAAH, M. Managerial social capital, strategic orientation, and organizational performance in an emerging economy. Strategic Management Journal, v. 28, p. $1235-$ 1255, 2007.

ADLER, P. S.; KWON, S. W. Social capital: prospect for a new concept. Academy of Management Review, v. 27, n. 1, p. 17-40, 2002.

ALBAGLI, S.; MACIEL, M. L. Capital social e empreendedorismo local. Projeto de pesquisa políticas para promoção de sistemas produtivos locais de MPME brasileiras. Rio de Janeiro: UFRJ/IE, 2002.

BARON, S.; FIELD, J.; SCHULLER, T. Social capital. Oxford: Oxford University Press, 2000.

BATJARGAL, B. Social capital and entrepreneurial performance in Russia: a longitudinal study. Organization Studies, v. 24, n. 4, p. 535-556, 2003.

BOURDIEU, P. Le capital social, notes provisoires. Actes de la recherche en sciences sociales, n. 31, 1980. p. 2-3. 
. Razões práticas: sobre a teoria da ação. 4 ed. Campinas: Papirus, 2003.

BROWN, E.; FERRIS, J. M. Social capital and philanthropy: an analysis of the impact of social capital on individual giving and volunteering. Nonprofit and Voluntary Sector Quarterly, v. 36, p. 85-99, 2007.

COLEMAN, J. S. Social capital in the creation of human capital. The American Journal of Sociology, v. 94, p. 95-120, 1988.

CROTTY, J. Reshaping the hourglass? The environmental movement and civil society development in the Russian Federation. Organization Studies, v. 27, n. 9, p. 13191338, 2006.

GRADDY, E.; WANG, L. Community foundation development and social capital. Nonprofit and Voluntary Sector Quarterly, v. 38, p. 392-412, 2009.

GOPALAKRISHNAN, S.; SCILLITOE, J. L.; SANTORO, M. D. Tapping deep pockets: the role of resources and social capital on financial capital acquisition by biotechnology firms in biotech-pharma alliances. Journal of Management Studies, v. 45, n. 8, p. 1354-1376, 2008.

ISHAM, J.; KOLODINSKY, J.; KIMBERLY, G. The effects of volunteering for nonprofit organizations on social capital formation: evidence from a statewide survey. Nonprofit and Voluntary Sector Quarterly, v. 35, p. 367-383, 2006.

LESTER, R. H. et al. Former government officials as outside directors: the role of human and social capital. Academy of Management Journal, v. 51, n. 5, p. 999-1013, 2008.

LUO, Y. Industrial Dynamics and Managerial Networking in an emerging market: the case of China. Strategic Management Journal, v. 24, p. 1315-1327, 2003.

MAURER, I.; EBERS, M. Dynamics of social capital and their performance implications: Lesson from Biotechnology Start-ups. Administrative Science Quarterly, v. 51, p. 262292, 2006.

MORAN, P. Structural vs. Relational embeddedness: social capital and managerial performance. Strategic Management Journal, v. 26, p. 1129-1151, 2005.

NAHAPIET, J. e GHOSHAL, S. Social capital, intellectual capital and the organizational advantage. Academy of Management Review, v. 23, n. 2, p. 242-266, 1998.

PIL, F. K.; LEANA, C. Applying organizational research to public school reform: the effects of teacher human and social capital on student performance. Academy of Management Journal, v. 52, n. 6, p. 1101-1124, 2009. 
PORTES, A. Social capital: its origins and applications in modern sociology. Annual Review of Sociology, v. 24, p. 1-24, 1998.

. The two meanings of social capital. Sociological Forum, v. 15, n. 1, p. 1-

$12,2000$.

PUTNAM, R. Bowling alone: America's declining social capital. Journal of Democracy, $v$. 6, n. 1, p. 65-78, 1995.

SARATE, J. A. R.; MACKE, J. Fatores explicativos do capital social em uma cidade da serra gaúcha: a percepção dos estudantes de Administração. In: ENANPAD, 31., 2007, Rio de Janeiro. Anais. Rio de Janeiro: Anpad, 2007. 1 CD.

SOMAYA, D.; WILLIAMSON, I. O.; LORINKOVA, N. Gone but not lost: the different performance impacts of employee mobility between cooperator versus competitors. Academy of Management Journal, v. 51, n. 5, p. 936-953, 2008.

STAM, W.; ELFRING, T. Entrepreneurial orientation and new venture performance: the moderating role of intra- and extraindustry social capital. Academy of Management Journal, v. 51, n. 1, p. 97-111, 2008.

STEVENSON, W. B.; RADIN, R. F. Social capital and social influence on the board of directors. Journal of Management Studies, v. 46, n. 1, p. 16-44, 2009.

XIAO, Z.; TSUI, A. S. When brokers may not work: the cultural contingency of social capital in chinese high-tech firms. Administrative Science Quarterly, v. 52, p. 1-31, 2007.

WALKER, E. Contingent pathways from joiner to activist: the indirect effect of participation in voluntary associations on civic engagement. Sociological Forum, v. 23, n. 1, p. 116-144, 2008.

WHITE, M. J.; GLICK, J. E. Generation status, social capital, and the routes out of high school. Sociological Forum, v. 15, n. 4, p. 671-691, 2000.

WU, W. Dimensions of social capital and Firm Competitiveness Improvement: the Mediating Role of Information Sharing. Journal of Management Studies, v. 45, n. 1, p. 122-146, 2008.

YEUNG, A. B. An Intricate Triangle - Religiosity, volunteering, and social capital: the european perspective, the case of Finland. Nonprofit and Voluntary Sector Quarterly, v. 33, p. 401-422, 2004. 
ASPECTOS METODOLÓGICOS DOS ESTUDOS EM CAPITAL SOCIAL: UMA ANÁLISE DOS PRINCIPAIS PERIÓDICOS INTERNACIONAIS

Rosana da Rosa Portella Tondolo - Vilmar Antonio Gonçalves Tondolo - Cláudia Cristina Bitencour

\section{APÊNDICE A}

\begin{tabular}{|l|l|l|l|l|l|}
\hline No & Journal & Título do Artigo & Autores & Ano & $\begin{array}{l}\text { Estratégias } \\
\text { Metodológicas }\end{array}$ \\
\hline 1 & Academy of & Former Government & LESTER, R. H. et al. & 2008 & Quantitativo - \\
\hline
\end{tabular}

R. eletr. estrat. neg., Florianópolis, v.5, n.1, p.110-136, jan./abr. 2012 


\section{ASPECTOS METODOLÓGICOS DOS ESTUDOS EM CAPITAL SOCIAL: UMA ANÁLISE DOS PRINCIPAIS PERIÓDICOS \\ INTERNACIONAIS}

Rosana da Rosa Portella Tondolo - Vilmar Antonio Gonçalves Tondolo - Cláudia Cristina Bitencour

\begin{tabular}{|c|c|c|c|c|c|}
\hline & $\begin{array}{l}\text { Management } \\
\text { Journal }\end{array}$ & $\begin{array}{llr}\text { officials as outside } & \text { a } \\
\text { directors: the role of } \\
\text { human and social } \\
\text { capital }\end{array}$ & & & $\begin{array}{l}\text { correlação e análise de } \\
\text { regressão testando } 2 \\
\text { modelos; } \\
\text { utilização de hipóteses. }\end{array}$ \\
\hline 2 & $\begin{array}{l}\text { Academy of } \\
\text { Management } \\
\text { Journal }\end{array}$ & $\begin{array}{l}\text { Gone but not lost: the } \\
\text { different performance } \\
\text { impacts of employee } \\
\text { mobility } \\
\text { cooperator between } \\
\text { competitors }\end{array}$ & $\begin{array}{l}\text { SOMAYA, } \\
\text { WILLIAMSON, I. O.; } \\
\text { LORINKOVA, N. }\end{array}$ & 2008 & $\begin{array}{l}\text { Quantitativo - } \\
\text { estatística descritiva, } \\
\text { correlação e análise de } \\
\text { regressão, modelo de } \\
\text { Poisson testando } 7 \\
\text { modelos; } \\
\text { utilização de hipóteses. }\end{array}$ \\
\hline 3 & $\begin{array}{l}\text { Academy of } \\
\text { Management } \\
\text { Journal }\end{array}$ & $\begin{array}{l}\text { Entrepreneurial } \\
\text { orientation and new } \\
\text { venture performance: } \\
\text { the moderating role of } \\
\text { intra- and extraindustry } \\
\text { social capital }\end{array}$ & $\begin{array}{ll}\text { STAM, } & \text { W.; } \\
\text { ELFRING, T. } & \end{array}$ & 2008 & $\begin{array}{l}\text { Quantitativo - } \\
\text { estatística descritiva, } \\
\text { correlação e análise de } \\
\text { regressão; } \\
\text { utilização de hipóteses. }\end{array}$ \\
\hline 4 & $\begin{array}{l}\text { Academy of } \\
\text { Management } \\
\text { Journal }\end{array}$ & $\begin{array}{l}\text { Applying organizational } \\
\text { research to public } \\
\text { school reform: the } \\
\text { effects of teacher } \\
\text { human and social } \\
\text { capital on student } \\
\text { performance }\end{array}$ & PIL, F. K.; LEANA, C. & 2009 & $\begin{array}{l}\text { Quantitativo - } \\
\text { correlação e Método de } \\
\text { regressão linear } \\
\text { hierárquica; } \\
\text { utilização de hipóteses. }\end{array}$ \\
\hline 5 & $\begin{array}{l}\text { Administra- } \\
\text { tive Science } \\
\text { Quarterly }\end{array}$ & $\begin{array}{l}\text { When brokers may not } \\
\text { work: the cultural } \\
\text { contingency of social } \\
\text { capital in chinese high- } \\
\text { tech firms }\end{array}$ & $\begin{array}{l}\text { XIAO, } \\
\text { TSUI, A. S. }\end{array}$ & 2007 & $\begin{array}{ll}\text { Quantitativo } & \text { e } \\
\text { Qualitativo - survey } & \text { e } \\
\text { entrevista; } & \\
\text { estatística descritiva e } \\
\text { correlação. }\end{array}$ \\
\hline 6 & $\begin{array}{l}\text { Administra- } \\
\text { tive Science } \\
\text { Quarterly }\end{array}$ & 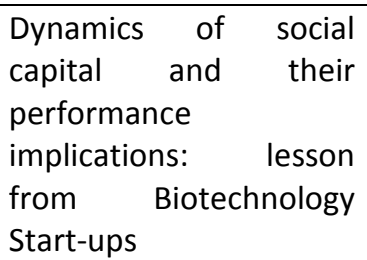 & $\begin{array}{l}\text { MAURER, I.; EBERS, } \\
\text { M. }\end{array}$ & 2006 & $\begin{array}{l}\text { Qualitativo - grounded } \\
\text { theory, entrevista. }\end{array}$ \\
\hline 7 & $\begin{array}{l}\text { Journal of } \\
\text { Management } \\
\text { Studies }\end{array}$ & $\begin{array}{l}\text { Dimensions of social } \\
\text { capital and Firm } \\
\text { Competitiveness } \\
\text { Improvement: } \\
\text { Mediating Role of } \\
\text { Information Sharing }\end{array}$ & WU, Wei-ping & 2008 & $\begin{array}{l}\text { Quantitativo e } \\
\text { Qualitativo - survey e } \\
\text { entrevistas; } \\
\text { estatística descritiva e } \\
\text { Correlação de Pearson; } \\
\text { utilização de hipóteses. }\end{array}$ \\
\hline 8 & $\begin{array}{l}\text { Journal of } \\
\text { Management } \\
\text { Studies }\end{array}$ & $\begin{array}{l}\text { Tapping deep pockets: } \\
\text { the role of resources } \\
\text { and social capital on } \\
\text { financial capital } \\
\text { acquisition by } \\
\text { biotechnology firms in } \\
\text { biotech-pharma } \\
\text { alliances }\end{array}$ & $\begin{array}{l}\text { GOPALAKRISHNAN, } \\
\text { S.; SCILLITOE, J. L.; } \\
\text { SANTORO, M. D. }\end{array}$ & 2008 & $\begin{array}{l}\text { Quantitativo - } \\
\text { estatística descritiva, } \\
\text { correlação e método de } \\
\text { regressão linear } \\
\text { hierárquica; } \\
\text { utilização de hipóteses. }\end{array}$ \\
\hline 9 & $\begin{array}{l}\text { Journal of } \\
\text { Management } \\
\text { Studies }\end{array}$ & $\begin{array}{l}\text { Social capital and social } \\
\text { influence on the board } \\
\text { of directors }\end{array}$ & $\begin{array}{l}\text { STEVENSON, W. B.; } \\
\text { RADIN, R. F. }\end{array}$ & 2009 & $\begin{array}{ll}\text { Quantitativo } & \mathrm{e} \\
\text { Qualitativo - survey } & \mathrm{e} \\
\text { entrevistas; } & \end{array}$ \\
\hline
\end{tabular}




\section{ASPECTOS METODOLÓGICOS DOS ESTUDOS EM CAPITAL SOCIAL: UMA ANÁLISE DOS PRINCIPAIS PERIÓDICOS INTERNACIONAIS \\ Rosana da Rosa Portella Tondolo - Vilmar Antonio Gonçalves Tondolo - Cláudia Cristina Bitencour}

\begin{tabular}{|c|c|c|c|c|c|}
\hline & & & & & $\begin{array}{l}\text { estatística descritiva e } \\
\text { Correlação de Pearson; } \\
\text { utilização de hipóteses. }\end{array}$ \\
\hline 10 & $\begin{array}{l}\text { Nonprofit } \\
\text { And } \\
\text { Voluntary } \\
\text { Sector } \\
\text { Quarterly }\end{array}$ & $\begin{array}{l}\text { An Intricate Triangle - } \\
\text { Religiosity, } \\
\text { volunteering, and social } \\
\text { capital: the European } \\
\text { perspective, the case of } \\
\text { Finland }\end{array}$ & YEUNG, A. B. & 2004 & $\begin{array}{l}\text { Quantitativo - dados } \\
\text { secundários; } \\
\text { tabelas cruzadas, ANOVA } \\
\text { e análise de regressão } \\
\text { logística. }\end{array}$ \\
\hline 11 & $\begin{array}{l}\text { Nonprofit } \\
\text { And } \\
\text { Voluntary } \\
\text { Sector } \\
\text { Quarterly }\end{array}$ & $\begin{array}{l}\text { The effects of } \\
\text { volunteering for } \\
\text { nonprofit organizations } \\
\text { on social capital } \\
\text { formation: evidence } \\
\text { from a statewide survey }\end{array}$ & $\begin{array}{ll}\text { ISHAM, } & \text { J.; } \\
\text { KOLODINSKY, } & \text { J.; } \\
\text { KIMBERLY, G. } & \end{array}$ & 2006 & $\begin{array}{l}\text { Quantitativo - dados } \\
\text { secundários; } \\
\text { modelo econométrico. }\end{array}$ \\
\hline 12 & $\begin{array}{l}\text { Nonprofit } \\
\text { And } \\
\text { Voluntary } \\
\text { Sector } \\
\text { Quarterly }\end{array}$ & $\begin{array}{l}\text { Community foundation } \\
\text { development and social } \\
\text { capital }\end{array}$ & $\begin{array}{l}\text { GRADDY, } \\
\text { WANG, L. }\end{array}$ & 2009 & $\begin{array}{l}\text { Quantitativo }- \text { dados } \\
\text { secundários; } \\
\text { estatística descritiva e } \\
\text { regressão ordinária do } \\
\text { quadrado mínimo. }\end{array}$ \\
\hline 13 & $\begin{array}{l}\text { Nonprofit } \\
\text { And } \\
\text { Voluntary } \\
\text { Sector } \\
\text { Quarterly }\end{array}$ & $\begin{array}{l}\text { Social capital and } \\
\text { philanthropy: an } \\
\text { analysis of the impact of } \\
\text { social capital on } \\
\text { individual giving and } \\
\text { volunteering }\end{array}$ & $\begin{array}{l}\text { BROWN, } \\
\text { FERRIS, J. M. }\end{array}$ & 2007 & $\begin{array}{l}\text { Quantitativo - dados } \\
\text { secundários; } \\
\text { estatística descritiva, } \\
\text { correlação e regressão. }\end{array}$ \\
\hline 14 & $\begin{array}{l}\text { Organization } \\
\text { Studies }\end{array}$ & $\begin{array}{l}\text { Reshaping the } \\
\text { hourglass? The } \\
\text { environmental } \\
\text { movement and civil } \\
\text { society development in } \\
\text { the Russian Federation }\end{array}$ & CROTTY, J. & 2006 & $\begin{array}{l}\text { Qualitativo - entrevistas; } \\
\text { método de triangulação } \\
\text { de dados. }\end{array}$ \\
\hline 15 & $\begin{array}{l}\text { Organization } \\
\text { Studies }\end{array}$ & $\begin{array}{l}\text { Social capital and } \\
\text { entrepreneurial } \\
\text { performance in Russia: } \\
\text { a longitudinal study }\end{array}$ & BATJARGAL, B. & 2003 & $\begin{array}{l}\text { Quantitativo - aplicação } \\
\text { de questionários; } \\
\text { estatística descritiva, } \\
\text { correlação de Pearson e } \\
\text { regressão multivariada. }\end{array}$ \\
\hline 16 & $\begin{array}{l}\text { Sociological } \\
\text { Forum }\end{array}$ & $\begin{array}{l}\text { Generation status, } \\
\text { social capital, and the } \\
\text { routes out of high } \\
\text { school }\end{array}$ & $\begin{array}{l}\text { WHITE, M. J.; } \\
\text { GLICK, J. E. }\end{array}$ & 2000 & $\begin{array}{l}\text { Quantitativo }- \text { dados } \\
\text { secundários; } \\
\text { modelo de regressão } \\
\text { logística e a regressão } \\
\text { logística multinomial. }\end{array}$ \\
\hline 17 & $\begin{array}{l}\text { Sociological } \\
\text { Forum }\end{array}$ & $\begin{array}{l}\text { Contingent pathways } \\
\text { from joiner to activist: } \\
\text { the indirect effect of } \\
\text { participation in } \\
\text { voluntary associations } \\
\text { on civic engagement }\end{array}$ & WALKER, E. T. & 2008 & $\begin{array}{l}\text { Quantitativo - dados } \\
\text { secundários; } \\
\text { equações estruturais. }\end{array}$ \\
\hline 18 & $\begin{array}{l}\text { Strategic } \\
\text { Management } \\
\text { Journal }\end{array}$ & $\begin{array}{lr}\text { Managerial } & \text { social } \\
\text { capital, } & \text { strategic } \\
\text { orientation, } & \text { and } \\
\text { organizational } & \\
\end{array}$ & ACQUAAH, M. & 2007 & 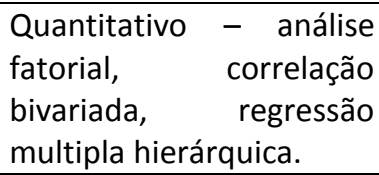 \\
\hline
\end{tabular}


ASPECTOS METODOLÓGICOS DOS ESTUDOS EM CAPITAL SOCIAL: UMA ANÁLISE DOS PRINCIPAIS PERIÓDICOS

INTERNACIONAIS

Rosana da Rosa Portella Tondolo - Vilmar Antonio Gonçalves Tondolo - Cláudia Cristina Bitencour

\begin{tabular}{|c|c|c|c|c|c|}
\hline & & $\begin{array}{l}\text { performance in an } \\
\text { emerging economy }\end{array}$ & & & \\
\hline 19 & $\begin{array}{l}\text { Strategic } \\
\text { Management } \\
\text { Journal }\end{array}$ & $\begin{array}{l}\text { Structural vs. Relational } \\
\text { embeddedness: social } \\
\text { capital and managerial } \\
\text { performance }\end{array}$ & MORAN, P. & 2005 & $\begin{array}{l}\text { Quantitativo - estatística } \\
\text { descritiva, correlação } \\
\text { bivariada e regressão } \\
\text { multivariada. }\end{array}$ \\
\hline 20 & $\begin{array}{l}\text { Strategic } \\
\text { Management } \\
\text { Journal }\end{array}$ & $\begin{array}{l}\text { Industrial Dynamics and } \\
\text { Managerial Networking } \\
\text { in an emerging market: } \\
\text { The case of China }\end{array}$ & LUO, Y. & 2003 & $\begin{array}{l}\text { Quantitativo - dados } \\
\text { secundários e primários } \\
\text { (survey); } \\
\text { estatística descritiva, } \\
\text { Correlação de Pearson, } \\
\text { regressão multivariada. } \\
\text { multinomial. }\end{array}$ \\
\hline
\end{tabular}

